Revue d'histoire de l'Amérique française

REVUE D.HISTOIRE DE L'AMÉRIQUE FRANÇAISE

\title{
La transmission du patrimoine dans les terroirs en expansion : un exemple canadien au XVIII ${ }^{\mathrm{e}}$ siècle
}

\section{Sylvie Dépatie}

Volume 44, numéro 2, automne 1990

URI : https://id.erudit.org/iderudit/304878ar

DOI : https://doi.org/10.7202/304878ar

Aller au sommaire du numéro

Éditeur(s)

Institut d'histoire de l'Amérique française

ISSN

0035-2357 (imprimé)

1492-1383 (numérique)

Découvrir la revue

Citer cet article

Dépatie, S. (1990). La transmission du patrimoine dans les terroirs en expansion : un exemple canadien au XVIII ${ }^{\mathrm{e}}$ siècle. Revue d'histoire de l'Amérique française, 44(2), 171-198. https://doi.org/10.7202/304878ar
Résumé de l'article

Cet article retrace le processus de transmission du patrimoine dans une seigneurie canadienne au XVIII ${ }^{\mathrm{e}}$ siècle. Il insiste sur la nécessité de tenir compte de la nature des biens transmis pour comprendre les formes et cerner les effets de ce processus. Cette approche permet de constater que la donation qui se généralise vers le milieu du XVIII ${ }^{\mathrm{e}}$ siècle n'implique pas nécessairement l'inégalité entre les enfants. De plus, elle indique que ce recours à la donation est davantage lié à l'accroissement de la capacité productive des exploitations qu'à la saturation du terroir. Par ailleurs, l'étude démontre que la présence de terres disponibles ne garantit pas l'efficacité du système de reproduction sociale et que ce système, quoique relativement égalitaire, permet le maintien des écarts économiques entre les générations.
Tous droits réservés @ Institut d'histoire de l'Amérique française, 1990
Ce document est protégé par la loi sur le droit d'auteur. L’utilisation des services d'Érudit (y compris la reproduction) est assujettie à sa politique d'utilisation que vous pouvez consulter en ligne.

https://apropos.erudit.org/fr/usagers/politique-dutilisation/ 


\title{
LA TRANSMISSION DU PATRIMOINE DANS LES TERROIRS EN EXPANSION: UN EXEMPLE CANADIEN AU XVIIIT SIÈCLE ${ }^{1}$
}

\author{
SYLVIE DÉPATIE \\ Département d'histoire \\ Université du Québec à Montréal
}

\section{RÉSUMÉ}

Cet article retrace le processus de transmission du patrimoine dans une seigneurie canadienne au XVIIIe siècle. Il insiste sur la nécessité de tenir compte de la nature des biens transmis pour comprendre les formes et cerner les effets de ce processus. Cette approche permet de constater que la donation qui se généralise vers le milieu du XVIIIe siècle n'implique pas nécessairement l'inégalité entre les enfants. De plus, elle indique que ce recours à la donation est davantage lié à l'accroissement de la capacité productive des exploitations qu'à la saturation du terroir. Par ailleurs, l'étude démontre que la présence de terres disponibles ne garantit pas l'efficacité du système de reproduction sociale et que ce système, quoique relativement égalitaire, permet le maintien des écarts économiques entre les générations.

\begin{abstract}
This article traces inheritance practices in an eighteenth century canadian seigneurie. It stresses the need to take into account the nature of the transmitted property in order to understand the forms and the effects of such transfers. This approach shows that the deed of gift, which became popular by the middle of the century, does not necessarily lead to inequality among the offspring. It also indicates that the use of the deed of gift has more to do with the productive capacity of the property than with the changing man-land ratio in the area. The study finally demonstrates that the availability of undevelopped land does not guarantee the success of the system of social reproduction and that, although relatively egalitarian, this system allows economic differences to be maintained from one generation to the next.
\end{abstract}

Cet article vise à cerner et à interpréter les modalités, les facteurs et les effets du processus de transmission du patrimoine dans une seigneurie canadienne au XVIIIe siècle.

Commencée à la fin du XVIIe siècle, la colonisation de l'île Jésus ne s'achève qu'à la fin du XVIIIe siècle. Par ailleurs, plusieurs seigneu-

\footnotetext{
1 Cet article reprend une partie de la démonstration de ma thèse de doctorat, L'évolution d'une société rurale: l'île Jésus au XVIIIe siècle, thèse de Ph.D, Université McGill,1988. Je remercie Mario Lalancette pour l'aide indispensable qu'il m'a accordée lors de la compilation des dossiers familiaux qui sont à la base de cette étude. Ma reconnaissance va également à Louise Dechêne, Louise Tremblay et Fernande Roy qui ont commenté une première version de ce texte.
} 
ries avoisinantes sont en même temps en voie de peuplement. La région constitue donc un bon terrain d'enquête pour cerner les caractéristiques de la reproduction familiale dans un terroir en expansion.

Mon travail s'inscrit dans la foulée de plusieurs études portant sur la transmission du patrimoine dans des régions québécoises de peuplement récent, que ce soit dans la vallée du Saint-Laurent ou celle du Richelieu aux XVIIe et XVIIIe siècles ou au Saguenay/Lac Saint-Jean à la fin du XIXe siècle. Ces enquêtes ont permis de dresser un bilan provisoire sur lequel s'appuie ma recherche.

\section{1 - LA TRANSMISSION DU PATRIMONE: LA THÉORIE ET L'ÉVOLUTION DE LA PRATIQUE AU CANADA DU XVIIe À LA FIN DU XIXe SIÈCLE}

Au Canada, avant 1760, la transmission des biens entre générations est régie par la Coutume de Paris qui prévoit la stricte égalité entre héritiers. Cependant, en respectant la réserve des quatre-quints et la légitime, un père peut favoriser un héritier par testament, dot ou donation entre vifs $^{2}$. Au moment du partage successoral, les enfants ne sont tenus de rapporter ce qu'ils ont reçu du vivant des parents que s'ils désirent participer au partage. Mais rien n'empêche un enfant favorisé de renoncer à la succession pour garder ce qu'il a déjà reçu ${ }^{3}$. La Coutume ellemême permet donc de déroger au principe de la stricte égalité entre héritiers.

Cependant, au Québec comme souvent ailleurs ${ }^{4}$, il y a une marge entre la règle juridique et les pratiques. Ainsi, il semble bien que, au stade initial du peuplement, soit, selon les régions, au XVIIe ou au XVIIIe siècle, les paysans canadiens se montrent plus égalitaires que ne l'exige la Coutume. La dot est rare et les testaments contiennent des dispositions à caractère spirituel plutôt que temporel. Quant à la donation, elle est peu fréquente et on y recourt le plus souvent avec l'accord de tous les héritiers dans le but d'assurer la subsistance de parents vieillisants. La plupart des successions donnent donc lieu à un partage égalitaire avec rapport dans les rares cas où il $\mathrm{y}$ a eu avance d'hoirie ${ }^{5}$.

2 La réserve des quatre-quints touche les biens propres, soit les immeubles échus à un des conjoints par succession en ligne directe ou collatérale et par donation en ligne directe. Cette réserve stipule que le de cujus ne peut disposer à sa guise que du cinquième de ses propres. La légitime touche tous les biens et correspond à la moitié de ce que les héritiers auraient reçu si le partage des biens avait été égalitaire. Y. Zoltvany, «Esquisse de la Coutume de Paris», Revue d'histoire de l'Amérique française, 25,3 (décembre 1971): 368, 381-382.

3 Ibid., 381. Allan Greer insiste avec raison sur les possibilités d'inégalité contenues dans cette disposition. A. Greer, Peasant, Lord and Merchant: Rural Society in Three Quebec Parishes 1740-1840 (Toronto, University of Toronto Press, 1985), 72-73.

4 Voir, par exemple, A. Collomp, «Alliances et filiation en Haute-Provence au XVIIIe siècle», Annales ESC, 32,3 (mai-juin 1977): 446.

5 Telles sont les pratiques observées par Louise Dechêne pour l'île de Montréal au XVIIe siècle. L. Dechêne, Habitants et marchands de Montréal au XVIIe siècle (Paris, Plon 1974), 294-298, 424-433. A. Greer affirme qu'il en va de même au début du XVIIIe siècle dans la vallée du Richelieu, op. cit., 74-80. 
Le constraste est saisissant entre cet égalitarisme initial et ce qui semble devenu courant dans la vallée du Saint-Laurent à la fin du XIXe siècle, dans un contexte de terroir plein, soit l'exclusion des enfants dotés et la transmission du patrimoine foncier à un seul héritier ${ }^{6}$.

Peut-on marquer les étapes de cette évolution? Selon Allan Greer qui a étudié le phénomène dans la vallée du Richelieu entre 1740 et 1840 , l'inégalité entre héritiers se manifeste dès le milieu du XVIIIle siècle. Alors que les partages post-mortem strictement égalitaires y sont la règle au début de la période de colonisation, ils disparaissent progressivement des greffes des notaires au profit de la donation entre vifs qui favorise un héritier au détriment des autres. De plus, Greer note que l'inégalité s'accentue au XIXe siècle, dans la mesure où la plupart des donations ne prévoient plus de compensations pour les cohéritiers désavantagés, comme c'était souvent le cas au XVIIIe siècle. Pour expliquer ce recours plus fréquent à la donation, Greer avance l'instauration de la liberté testamentaire en 1774, le développement du marché des grains qui rend plus important le maintien d'exploitations assez grandes pour produire des surplus et le resserrement du rapport hommes/terres ${ }^{7}$.

Paradoxalement, Gérard Bouchard présente la donation comme une des formes de transmission typiques d'un «système ouvert» de reproduction familiale. Défini à partir de l'observation des pratiques de transmission au Saguenay/Lac Saint-Jean à la fin du XIXe siècle, ce système ouvert s'oppose aux systèmes clos des terroirs pleins. À la différence de ces derniers qui excluent certains descendants dans le but de préserver l'intégrité du patrimoine, il comporte plutôt des stratégies visant à étendre les assises foncières de la famille afin d'établir le plus d'enfants possible. Plutôt que de se limiter à une opération «dramatique et ponctuelle» au moment de la succession, la transmission en système ouvert prend surtout des formes non successorales s'étalant sur plusieurs décennies.

Selon Bouchard, deux types de stratégies permettent d'atteindre l'objectif de ce système ouvert. La première implique la migration de familles entières à partir de vieux terroirs vers des zones de colonisation où ces familles acquièrent à bas prix suffisamment de terre pour établir tous leurs fils. La seconde, plus complexe, comporte trois phases distinctes:

1 - Attribution aux fils mariés de lots acquis par la famille et partiellement défrichés (les filles ne reçoivent pas de terre, on essaie de les marier à des garçons eux-mêmes établis);

2 - Attribution à un fils, qui est rarement l'aîné, du «vieux bien» [...];

6 L. Gérin, «L'habitant casanier au coeur de la vallée laurentienne», Le type économique et social des Canadiens. Milieux agricoles de tradition française (Montréal, Fides, 1948), 77-78.

A. Greer, op. cit., 74-81. 
3 - Attribution des biens subsistants - surtout mobiliers - au moment du décès du dernier conjoint ${ }^{8}$.

Ces pratiques seraient particulièrement efficaces puisqu'elles permettrait d'établir près de trois enfants sur quatre comme agriculteurs. Cependant, elles seraient essentiellement conditionnées par la présence de terres disponibles, si bien que, dès la disparition de ce facteur, elles entreraient en mutation vers les stratégies défensives des systèmes $\operatorname{clos}^{9}$.

L'historiographie actuelle considère donc la densité de la population rurale comme le facteur explicatif fondamental non seulement des formes mais également de l'orientation et de l'efficacité du système de reproduction social de la paysannerie québécoise du XVIIe à la fin du XIXe siècle. Avec la saturation progressive et successive des terroirs, on passerait d'un système égalitaire, axé sur l'établissement des enfants et très efficace à un système inégalitaire, axé sur la préservation du patrimoine familial et, partant, générant des exclus.

\section{2 - PROBLÉMATIQUE ET MÉTHODOLOGIE}

Ce modèle est bien sûr séduisant par sa logique. Cependant, il demeure fragile en raison de lacunes théoriques et méthodologiques. Trois aspects, en particulier, font problème.

Le premier est la confusion entretenue entre les modalités de la transmission, le degré d'égalité et le degré d'efficacité. Par exemple, il apparaît imprudent de conclure à l'évolution vers l'inégalité à partir de la seule observation de la fréquence des donations dans les greffes notariés. Ainsi, à l'île Jésus, comme dans la vallée du Richelieu, le XVIIIe siècle est marqué par le recours de plus en plus fréquent à la donation. Complètement absent des greffes notariés de l'île avant 1730 , ce type d'acte devient très fréquent dans les décennies subséquentes. Cependant, la multiplication des donations ne marque pas la disparition des partages (tableau 1) et elle ne signifie pas nécessairement l'adoption de pratiques inégalitaires. Un examen attentif du contenu des partages et des donations et une bonne connaissance des patrimoines familiaux montrent en effet que ces actes ne concernent souvent qu'une partie des biens fonciers d'un couple et que le reste peut faire l'objet d'un autre mode de transmission. C'est le cas de la majorité des partages et des donations que j'ai examinés ${ }^{10}$. Donation

8 G. Bouchard, «Sur la reproduction familiale en milieu rural: systèmes ouverts et systèmes clos», Recherches sociographiques, 28,2-3 (1987): 230. Voir aussi, "Les systèmes de transmission des avoirs familiaux et le cycle de la société rurale au Québec, du XVIIe au XXe siècle», Histoire sociale/Social History, 16,31 (mai 1981): 35-60.

9 G. Bouchard, "Sur la reproduction familiale...», loc. cit., 239 et 245.

10 Sur 62 partages faits entre 1730 et 1774,51 ne concernent qu'une partie du patrimoine foncier soit le plus souvent la part du conjoint prédécédé. Il en va de même pour 75 donations sur 116 rédigées entre 1730 et 1775 . 
et partage ne s'excluent donc pas l'un l'autre, pas plus d'ailleurs qu'ils n'excluent le recours à d'autres stratégies de transmission comme la donation en avance d'hoirie de terres additionnelles ou de biens meubles. Par contre, l'utilisation de ces formes non successorales de transmission ne doit pas non plus être interprétée comme un signe d'égalité ou un gage d'efficacité car, comme nous le verrons, tout dépend de ce qui est transmis par ces procédés.

TABLEAU 1

Nombre de donations et de partages par décennie dans le greffe du notaire C.-F. Coron (1734-1767)

\begin{tabular}{lccc}
\hline Décennie & Partages & Donations & Total \\
\hline $1734-1739$ & 12 & 4 & 16 \\
$1740-1749$ & 20 & 17 & 37 \\
$1750-1759$ & 14 & 53 & 67 \\
$1760-1767$ & 13 & 41 & 54 \\
Total & 59 & 115 & 174 \\
\hline
\end{tabular}

Source: ANQM, Greffe du notaire Charles-François Coron.

Le second problème de l'historiographie actuelle tient au fait qu'on déduit l'orientation du système de reproduction de l'observation des pratiques de transmission de l'ensemble de la paysannerie, sans tenir compte des inégalités socio-économiques qui s'y rencontrent. Or, bon nombre de familles paysannes font, en matière de transmission du patrimoine, davantage ce qu'elles peuvent que ce qu'elles veulent. Pour cerner les buts du système, il faut donc observer la stratégie des couples qui, en raison de leur aisance économique, ont vraiment été en mesure d'effectuer un choix lors de la transmission de leurs biens.

Enfin, le troisième problème de l'historiographie découle de la notion d'efficacité du système de reproduction. Pour la mesurer, Gérard Bouchard a recours aux déclarations professionnelles contenues dans les registres paroissiaux et les recensements. Ces déclarations sont utilisées comme «marqueurs de l'établissement foncier», un individu déclarant la profession d'agriculteur étant considéré comme établi ${ }^{11}$. Cette méthode mesure donc l'efficacité du système en fonction de l'accès des enfants à la propriété foncière, quel que soit le rôle de la famille dans l'acquisition de la terre et quel que soit l'état de la terre. Sont considérés comme établis tant ceux qui possèdent une terre peu défrichée que ceux qui sont à la tête d'une exploitation dont la mise en valeur est avancée. Les premiers, bien sûr, sont propriétaires, mais il m'apparaît abusif de qualifier ce résultat de reproduction familiale réussie.

11 G. Bouchard, «Sur la reproduction familiale...», loc. cit., 235. Dans le cas des filles, on considère comme établies celles dont le mari est lui-même agriculteur, ibid., 239. 
Il me semble en effet que l'efficacité d'un système de reproduction se mesure plutôt à sa capacité d'assurer aux héritiers un point de départ leur permettant d'atteindre éventuellement une position socioéconomique au moins équivalente à celle des parents, ce qui n'est certes pas le cas lorsque la famille quitte une bonne exploitation dans une vieille paroisse pour installer ses fils sur des terres de colonisation ou lorsqu'un couple transmet à un ou plusieurs fils des terres non défrichées en réservant à un seul l'exploitation principale.

Ces considérations débouchent sur deux impératifs méthodologiques. D'une part, puisque la transmission du patrimoine est un processus long et complexe qui commence quelquefois peu après le mariage des parents pour se terminer longtemps après leur décès,il convient, pour la comprendre, d'examiner non seulement les formes successorales de transmission mais aussi l'ensemble des gestes posés par le couple pour installer ses héritiers: achat de terre, dot, donation en avancement d'hoirie, donation à rente, sans oublier les formes d'établissement non foncier comme la mise en apprentissage et, plus tard au XIXe siècle, le paiement d'études. En fait, c'est l'évolution économique de la cellule familiale sur plusieurs décennies qu'il faut reconstituer. D'autre part, pour vraiment cerner le degré d'égalité de même que l'orientation du système de reproduction, il faut connaître la nature des biens transmis. Or, les actes notariés sont les seules sources qui permettent de satisfaire à cette double exigence. Dès lors, leur utilisation est indispensable si on veut comprendre l'orientation des pratiques familiales et les facteurs qui les conditionnent ${ }^{12}$.

Aux fins de cette enquête, $\mathrm{j}$ 'ai donc reconstitué les pratiques de transmission de 118 ménages paysans de l'île Jésus ayant transmis leur patrimoine entre 1720 et 1775 . Ces familles ont fait l'objet d'une reconstitution démographique et, pour chacune, $\mathrm{j}$ 'ai tenté de suivre l'évolution du domaine foncier et les formes tant successorales que non successorales de la transmission. Ce groupe de familles-témoins a été constitué à partir des communautés ayant donné lieu à un inventaire après décès ou ayant fait donation pendant la période, en fonction de la possibilité de reconstituer l'ensemble du processus de transmission à partir des actes contenus dans les greffes des notaires de la région. En conséquence, le groupe à l'étude est entièrement composé de ménages formés avant 1750 et les couples qui se sont mariés après 1730 y sont moins bien représentés que ceux qui se sont unis avant cette date ${ }^{13}$.

12 Je rejoins ici l'opinion de Claire Dolan qui s'appuie sur ses propres recherches et sur les travaux de Pierre Lamaison et d'Alain Collomp, «L'image du père en Provence au XVIe siècle», Histoire Sociale/Social History, 18,35 (mai 1985): 31.

13 La reconstitution démographique a été effectuée à partir des registres paroissiaux des trois paroisses qui existaient alors à l'île Jésus, complétés par les dictionnaires généalogiques Tanguay et Jetté, ainsi que par les renseignements fournis par les actes notariés eux-mêmes. C. Tanguay, Dictionnaire généalogique des familles canadiennes-françaises (Montréal, Éditions Élysée, 1975, réédition), 7 vol.; R. Jetté, Dictionnaire généalogique des familles du Québec (Montréal, Presses de l'Université de Montréal, 1983, 1176 p.). Je remercie le Programme de démographie historique de l'Université de Montréal qui m'a fourni la transcription des registres paroissiaux. 


\section{3 - LES MODALITÉS DE LA TRANSMISSION}

Quelles sont les principales formes de transmission utilisées au XVIIIe siècle par les paysans de l'île Jésus? Le choix de celles-ci évolue-t-il avec le temps et, si oui, est-ce vers l'inégalité? Je réponds à ces questions en examinant premièrement comment les 118 ménages étudiés ont transmis leur exploitation principale ${ }^{14}$.

TABLEAU 2

Mode de transmission de l'exploitation principale

(118 ménages paysans)

1720-1775

\begin{tabular}{|c|c|c|c|c|c|}
\hline \multicolumn{5}{|c|}{ Mode de transmission } & \multirow[t]{2}{*}{ Total } \\
\hline $\begin{array}{l}\text { Partage } \\
\text { de toute } \\
\text { la terre }\end{array}$ & $\begin{array}{l}\text { Partage et } \\
\text { donation de } \\
\text { la part du } \\
\text { conjoint } \\
\text { survivant }\end{array}$ & $\begin{array}{l}\text { Partage et } \\
\text { vente de } \\
\text { la part du } \\
\text { conjoint } \\
\text { survivant }\end{array}$ & $\begin{array}{l}\text { Donation } \\
\text { de toute } \\
\text { la terre }\end{array}$ & Renonciation & \\
\hline 20 & 49 & 6 & 42 & 1 & 118 \\
\hline
\end{tabular}

Sources: Actes notariés et fichier des ménages paysans.

\section{1 - La transmission de l'exploitation principale}

Le tableau 2 illustre la situation. Soixante-quinze des 118 successions examinées ont donné lieu à un partage. À la suite de la dissolution de la communauté par le décès d'un des parents, la terre a été divisée également entre le conjoint survivant et les héritiers et le lot de ces derniers a été partagé en autant de parts qu'il y avait d'enfants. Les bâtiments et les biens meubles ont été partagés de la même façon. Ce partage a pu prendre place immédiatement après le décès, mais il a pu n'intervenir qu'à l'arrivée à l'âge adulte d'un des héritiers ou au remariage du conjoint survivant. S'il n'y a pas de remariage, l'indivision persiste parfois jusqu'au décès du conjoint survivant. L'ensemble de l'exploitation est alors divisée également entre les héritiers.

Généralement, cependant, le partage est fait en deux temps, soit après la mort de chacun des conjoints. Les modalités de partage de la part du dernier décédé varient selon son état matrimonial. S'il ne s'est pas remarié, sa part est divisée également entre les enfants. S'il s'est remarié, les règles de partage dépendent des clauses de son contrat de mariage. Sauf rares exceptions, il a gardé sa part en propre et celle-ci fait alors l'objet d'un partage égalitaire entre tous ses enfants, quel que soit le lit dont ils sont issus. Assez souvent cependant, le conjoint

14 L'exploitation principale comprend la terre de la communauté qui a le plus de valeur et les bâtiments qui y sont construits. Le ménage réside presque toujours sur cette terre et, généralement, les biens meubles de la communauté sont compris dans le processus de transmission de l'exploitation principale. 
remarié a, par son contrat de mariage, fait don à son futur conjoint «de pareille part et portion que chacun de ses enfants pourra amender de ses biens venant à sa succession future pour en jouir en toute propriété». La part est alors divisée en autant de portions qu'il y a d'enfants et une portion est ajoutée pour le conjoint survivant du deuxième mariage.

La suite de ces partages est toujours la même: il y a rachat des parts successorales foncières par un individu, le plus souvent un fils, quelquefois un gendre, un beau-père, voire un étranger à la famille. Ce processus de remembrement peut s'étaler sur vingt ans et donne parfois lieu à une compétition entre deux ou trois acheteurs.

Le partage intégral de la totalité de l'exploitation de la communauté est évidemment la forme de transmission la plus égalitaire. Tous les héritiers, quel que soit leur sexe, reçoivent une part égale dans l'héritage. Cette forme de transmission est cependant peu courante puisque seulement 20 des 118 successions analysées ont été réglées de cette façon. Plus fréquemment, lorsqu'il y a partage entre les héritiers et le conjoint survivant, celui-ci dispose de sa part avant son décès.

Exceptionnellement, les immeubles dévolus au conjoint survivant font l'objet d'une vente à un étranger. Cette pratique est surtout le fait de veuves remariées dont les enfants sont arrivés à l'âge adulte. Dans tous les cas observés, ceux-ci vendent leurs parts successorales au même acheteur avant ou après la transaction de leur mère.

Beaucoup plus fréquemment, le conjoint survivant donne sa part de l'exploitation à rente viagère. Cette façon de procéder est d'ailleurs le mode de transmission le plus fréquent: 49 des 118 successions se règlent de cette façon. La donation est parfois faite avant ou au moment du partage mais, généralement, elle intervient après celui-ci, lorsque le conjoint survivant est assez âgé (tableau 3). Cette façon de procéder est surtout le fait de veuves. Le donataire est le plus souvent un fils, récemment marié ou à la veille de son mariage. Dans plus de la moitié des cas où c'est un fils qui reçoit la donation, le choix se porte sur le fils aîné, mais il ne faut sans doute pas y voir une règle de primogéniture (tableau 4). En effet, un autre facteur oriente le choix du donataire: celui-ci a souvent déjà commencé à racheter les parts successorales sur la portion du conjoint décédé et il apparait donc logique de lui donner le reste de l'exploitation en donation. Amorcé avant ou après la donation, le remembrement de l'ensemble de la terre par le donataire est en effet le dénouement le plus fréquent de cette forme de transmission qui combine partage et donation.

En assurant le partage égal de la part d'un des conjoints, celle-ci respecte l'exigence de la légitime et, théoriquement, laisse le donateur libre de disposer de sa part sans prévoir de compensations pour les hé- 


\section{TABLEAU 3}

Caractéristiques des donateurs et des donataires pour les donations touchant la part du conjoint survivant (49 donations)

1720-1775

\section{DONATEUR}

(49)

Sexe

Masculin : 18

Féminin : 31

Statut matrimonial

Veuf : 33

Âge moyen

Homme : 58.6 ans (15 cas)

Femme : 56.5 ans $(23$ cas $)$

\section{DONATAIRE}

(51)

Lien de parenté

$\begin{array}{lll}\text { Fils } & : & 31^{*} \\ \text { Gendre } & : & 8^{*} \\ \text { Tous les enfants } & : & 4 \\ \text { Étranger } & : & 7\end{array}$

Âge moyen

20.7 ans

: 34 cas

Statut matrimonial

Célibataire : 13

Marié $\quad: 24$

Inconnu $\quad: 14$

Délai mariage/donation

$\begin{array}{ll}\text { Célibataire } & : 1.8 \text { ans } \\ \text { Marié } & : 5.3 \text { ans }\end{array}$

* Une donation faite à 2 fils et une à un fils et un gendre.

Sources: 49 donations à rente viagère et fichier des ménages paysans.

\section{TABLEAU 4}

Rang du donataire selon le nombre d'enfants mâles pour les donations touchant la part du conjoint survivant

\begin{tabular}{|c|c|c|c|c|c|c|c|c|}
\hline \multirow{2}{*}{$\begin{array}{l}\text { Nombre } \\
\text { d'enfants } \\
\text { mâles }\end{array}$} & \multicolumn{7}{|c|}{ Rang du donataire } & \multirow[t]{2}{*}{ Total } \\
\hline & 1 & 2 & 3 & 4 & 5 & 6 & 7 & \\
\hline 1 & 3 & & & & & & & 3 \\
\hline 2 & 1 & 3 & & & & & & 4 \\
\hline 3 & 6 & 2 & 2 & & & & & 10 \\
\hline 4 & 4 & 1 & 1 & & & & & 6 \\
\hline 5 & 2 & & & 1 & 2 & & & 5 \\
\hline 6 & & & & & & & & \\
\hline 7 & 1 & & & & & & & 1 \\
\hline 8 & & 2 & & & & & & 2 \\
\hline Total & 17 & 8 & 3 & 1 & 2 & & & 31 \\
\hline
\end{tabular}

Sources: Donations à rente viagère et fichier des ménages paysans. 
ritiers désavantagés. Cependant 22 des 49 donations prévoient des légitimes pour ces derniers. Quelquefois (6 cas), le donateur réserve la moitié de sa part de terre comme légitime pour l'ensemble des héritiers désavantagés. Plus rarement ( 2 cas $)$, il y ajoute une légitime en argent mais, le plus souvent (14 cas), les légitimes sont stipulées exclusivement en argent. Il est alors difficile de déterminer si celles-ci représentent la moitié de la valeur des biens donnés à rente. Cependant, à trois exceptions près, elles sont trop considérables pour n'être que symboliques.

Moins égalitaire que le partage intégral, la combinaison partagedonation assure aux héritiers de toucher au moins la moitié de ce qu'ils auraient reçu en cas de partage intégral et, souvent même, elle leur procure plus que ne le requiert la Coutume. En autant qu'on puisse en juger, ce serait la situation dans presque la moitié des cas observés.

Qu'en est-il maintenant des cas où les parents ont disposé de l'exploitation par donation avant leur décès? C'est la situation dans 42 des 118 successions analysées. Le recours exclusif à la donation est donc presque aussi fréquent que l'utilisation combinée du partage et de la donation et il est beaucoup plus courant que le partage intégral de toute l'exploitation.

Les couples qui choisissent ce mode de transmission sont arrivés à l'âge de la retraite comme en témoigne l'âge moyen du mari au moment de la donation qui est de 60 ans (tableau 5). Ils choisissent le plus souvent un, quelquefois deux, de leurs fils comme donataires. Exceptionnellement, un gendre ou un étranger à la famille est le bénéficiaire de la donation ${ }^{15}$. Le donataire choisi est le plus souvent un jeune célibataire à la veille de se marier. Dans plus du tiers des cas, il s'agit du plus vieux des garçons mais cette proportion n'est pas suffisante pour conclure que les aînés bénéficient d'une préférence (tableau 6). Le choix du donataire apparaît plutôt fondé sur une conjonction d'intérêts entre les donateurs prêts à se retirer et un fils prêt à prendre la relève.

En effet, pour les cédants, la donation semble davantage un moyen d'assurer leur retraite qu'un procédé pour exclure les non-donataires. $\mathrm{La}$ fréquente mention de légitimes dans les donations en constitue la preuve. Parfois, la légitime prend la forme de la réserve de la moitié de la terre aux non-donataires (8 cas). D'autres donations (6 cas) prévoient des compensation en terre pour certains héritiers et en argent pour les autres. Plus souvent toutefois (20 cas), les légitimes sont fixées exclusivement en argent.

15 Le choix d'un étranger à la famille est visiblement une solution de rechange qui n'intervient qu'en l'absence d'un fils ou à la suite de l'annulation d'une première donation faite à un fils. 


\section{TABLEAU 5}

Caractéristiques des donateurs et des donataires pour les donations touchant toute la terre principale (42 donations)

1720-1775

\section{DONATEUR}

(42)

Âge moyen

60.2 ans (39 cas)

\section{DONATAIRE}

(50)

Lien de parenté

$\begin{array}{ll}\text { Fils } & : 40^{*} \\ \text { Gendre } & : 6^{*} \\ \text { Tous les héritiers } & : 1 \\ \text { Étranger } & : 3 \\ 24.5 \text { ans } & : 36 \text { cas } \\ \text { Célibataire } & : 29 \\ \text { Marié } & : 16 \\ \text { Inconnu } & : 5 \\ \text { Célibataire } & : 1.9 \text { ans } \\ \text { Marié } & : 5.0 \text { ans }\end{array}$

Âge moyen

Statut matrimonial

Marié

: 5.0 ans

* Une donation à 2 fils, 1 donation à 1 fils et 1 gendre et 1 donation à 2 gendres.

Sources: 42 donations à rente viagère et fichier des ménages paysans.

\section{TABLEAU 6}

Rang du donataire selon le nombre d'enfants mâles pour les donations touchant toute la terre principale

\begin{tabular}{|c|c|c|c|c|c|c|c|c|c|}
\hline \multirow{2}{*}{$\begin{array}{l}\text { Nombre } \\
\text { d'enfants } \\
\text { mâles }\end{array}$} & \multicolumn{8}{|c|}{ Rang du donataire } & \multirow[t]{2}{*}{ Total } \\
\hline & 1 & 2 & 3 & 4 & 5 & 6 & 7 & 8 & \\
\hline 1 & 3 & & & & & & & & 3 \\
\hline 2 & 7 & 4 & & & & & & & 11 \\
\hline 3 & 2 & 2 & 5 & & & & & & 9 \\
\hline 4 & 3 & 1 & 1 & 2 & & & & & 7 \\
\hline 5 & & 1 & & & & & & & 1 \\
\hline 6 & & & & 1 & 1 & 1 & & & 3 \\
\hline 7 & & & & & 2 & 1 & 1 & & 4 \\
\hline 8 & & & & & & & 2 & & 2 \\
\hline Total & 15 & 8 & 6 & 3 & 3 & 2 & 3 & & 40 \\
\hline
\end{tabular}

Sources: Donations à rente viagère et fichier des ménages paysans. 
J'ai observé quelques donations où la légitime accordée dans la donation dépassait le montant requis pour la légitime légale. Mais il n'est pas toujours facile d'évaluer à quoi correspondent les sommes offertes aux héritiers non favorisés parce que la plupart du temps, la valeur du bien donné à rente n'est pas connue exactement. Toutefois, comme c'est le cas dans les donations de part de conjoint survivant, la plupart des donations ( 23 cas sur 26) prévoient des sommes trop importantes pour n'être que symboliques. Aux légitimes fixées en terre ou en argent, s'ajoutent couramment des dons en bétail et en meubles. Quelquefois, on précise aussi que le donataire devra nourrir et entretenir ses soeurs «à son pot et feu» jusqu'à leur mariage et même on l'oblige à fournir des vivres à un frère pendant que celui-ci défriche sa terre ${ }^{16}$.

La donation ne signifie donc pas toujours une dérogation à la Coutume et l'exclusion totale des enfants défavorisés. Seulement le quart des donations privent les cohéritiers de toute part dans le patrimoine familial. Elles sont généralement faites avec l'assentiment des nondonataires qui agissent ainsi en invoquant «leur devoir en qualité de bons enfants», envers des parents démunis ${ }^{17}$. Alors qu'elle est théoriquement la forme la plus inégalitaire de transmission, la donation permet donc aux héritiers de toucher au moins la légitime légale dans la majorité des cas analysés.

L'analyse qui précède révèle qu'à l'île Jésus entre 1720 et 1775 , le partage intégral est minoritaire et que la donation en tout ou en partie de l'exploitation principale constitue la forme de transmission la plus courante. Cette situation est-elle le fruit d'une évolution analogue à celle perçue par Allan Greer dans la vallée du Richelieu, soit le passage du recours majoritaire au partage au début du XVIIIe siècle à l'utilisation de plus en plus fréquente de la donation dans les décennies subséquentes ${ }^{18}$ ? Le classement des modes de transmission selon la période du mariage ne permet pas de réellement trancher la question (tableau 7). À mesure qu'on avance dans le XVIIIe siècle, il semble y avoir une légère tendance à délaisser les partages mais les résultats ne sont guère concluants. Plus nette est l'influence de la durée de l'union sur le mode de transmission pratiquée (tableau 8). Les partages interviennent plus fréquemment dans le cas d'unions prématurément brisées et, à mesure que le mariage se prolonge, les parents ont de plus en plus tendance à avoir recours à la donation ${ }^{19}$. En soi, la chose apparaît

16 ANQM, M.not. C.-F. Coron, 21 octobre 1760, Donation à rente par Jean Gravelle et Barbe Chartrand à Jacques Gravelle.

17 ANQM, M.not. C.-F. Coron, 27 mars 1753, Donation de Jacques Desnoyers et de Marie Noël à François Desnoyers.

18 A. Greer, op. cit., 74.

19 La situation est encore plus nette que ne le montre le tableau 8 puisque, dans les 19 donations faites par des couples pour lesquels il n'a pas été possible d'établir la date du décès du premier décédé, 18 ont été faites après au moins 20 ans de mariage. 
TABLEAU 7

Mode de transmission de l'exploitation principale selon la date du mariage

(118 ménages paysans)

1720-1775

\begin{tabular}{|c|c|c|c|c|c|c|}
\hline \multirow{2}{*}{$\begin{array}{l}\text { Période du } \\
\text { mariage }\end{array}$} & \multicolumn{5}{|c|}{ Mode de transmission } & \multirow[t]{2}{*}{ Total } \\
\hline & $\begin{array}{l}\text { Partage de } \\
\text { toute la } \\
\text { terre }\end{array}$ & $\begin{array}{l}\text { Partage et } \\
\text { donation de } \\
\text { la part du } \\
\text { conjoint } \\
\text { survivant }\end{array}$ & $\begin{array}{l}\text { Partage et } \\
\text { vente de } \\
\text { la part du } \\
\text { conjont } \\
\text { survivant }\end{array}$ & $\begin{array}{l}\text { Donation de } \\
\text { toute la } \\
\text { terre }\end{array}$ & Renonciation & \\
\hline Avant 1700 & 2 & 3 & 1 & & & 6 \\
\hline $1700-1709$ & 1 & 6 & 1 & 5 & & 13 \\
\hline 1710-1719 & 8 & 9 & 1 & 6 & & 24 \\
\hline $1720-1729$ & 2 & 16 & 3 & 18 & & 39 \\
\hline $1730-1739$ & 5 & 11 & & 10 & & 26 \\
\hline $1740-1749$ & 2 & 4 & & 3 & 1 & 10 \\
\hline Total & 20 & 49 & 6 & 42 & 1 & 118 \\
\hline
\end{tabular}

Sources: Actes notariés et fichier des ménages paysans.

\section{TABLEAU 8}

Mode de transmission de l'exploitation principale selon la durée de l'union

(118 ménages paysans) 1720-1775

\begin{tabular}{|c|c|c|c|c|c|c|}
\hline \multirow{2}{*}{$\begin{array}{l}\text { Durée de } \\
\text { l'union }\end{array}$} & \multicolumn{5}{|c|}{ Mode de transmission } & \multirow[t]{2}{*}{ Total } \\
\hline & $\begin{array}{l}\text { Partage de } \\
\text { toute la } \\
\text { terre }\end{array}$ & $\begin{array}{l}\text { Partage et } \\
\text { donation de } \\
\text { la part du } \\
\text { conjoint } \\
\text { survivant }\end{array}$ & $\begin{array}{l}\text { Partage et } \\
\text { vente de } \\
\text { la part du } \\
\text { conjont } \\
\text { survivant }\end{array}$ & $\begin{array}{l}\text { Donation de } \\
\text { toute la } \\
\text { terre }\end{array}$ & Renonciation & \\
\hline $0-9$ ans & 5 & 2 & & & & 7 \\
\hline $10-19$ ans & 11 & 13 & 3 & & 1 & 28 \\
\hline $20-29$ ans & 1 & 18 & 2 & 5 & & 26 \\
\hline $30-39$ ans & 1 & 11 & & 6 & & 18 \\
\hline $\begin{array}{l}40 \text { ans } \\
\text { et plus }\end{array}$ & 2 & 4 & & 12 & & 18 \\
\hline Inconnue & & 1 & 1 & 19 & & 21 \\
\hline Total & 20 & 49 & 6 & 42 & 1 & 118 \\
\hline
\end{tabular}

Sources: Actes notariés et fichier des ménages paysans. 
comme un truisme. Les couples comptant moins de 20 ans de vie conjugale ne songent pas à la retraite et n'ont pas d'enfants en âge de recevoir une donation, si bien que lorsque la mort interrompt leur union, leurs biens sont partagés. À l'inverse, les couples âgés aspirent au repos et ils ont des héritiers aptes à leur succéder. Ce qu'il faut néanmoins retenir de cette situation, c'est que la tendance à recourir à la donation est sans doute encore plus forte que ne l'indique le tableau 8. On peut en effet présumer que, si leur union s'était prolongée, les couples brisés prématurément auraient eux aussi disposé de leurs biens avant leur décès. La chose est d'autant plus plausible qu'ils auraient, au cours de leur mariage, davantage eu la possibilité d'accumuler des terres additionnelles pour installer des héritiers ne bénéficiant pas de la donation de l'exploitation principale.

\section{2 - La transmission des terres additionnelles}

L'analyse du mode de transmission de l'exploitation principale ne suffit pas, en effet, à cerner l'ensemble du processus de transmission car certains couples ont plus d'une terre à transmettre. Sur les 118 ménages analysés, 47 étaient dans cette situation (tableau 9).

TABLEAU 9

Répartition des ménages

selon le nombre de terres transmises

(118 ménages paysans)

1720-1775

\begin{tabular}{ccc}
\hline $\begin{array}{c}\text { Nombre } \\
\text { de terres } \\
\text { transmises }\end{array}$ & $\begin{array}{c}\text { Nombre de } \\
\text { ménages }\end{array}$ & $\begin{array}{c}\text { Total des } \\
\text { terres } \\
\text { transmises }\end{array}$ \\
\hline 1 & 71 & 71 \\
2 & 23 & 46 \\
3 & 14 & 42 \\
4 & 4 & 16 \\
5 & 2 & 10 \\
6 & 2 & 12 \\
7 & 1 & 7 \\
8 & - & - \\
9 & 1 & 9 \\
Total & 118 & 213 \\
\hline
\end{tabular}

Sources: Actes notariés et fichier des ménages paysans.

Dans une minorité de cas, ces «ajouts« sont transmis en même temps, de la même façon et aux mêmes bénéficiaires que l'exploitation principale (tableau 10). Il s'agit alors généralement de terres peu défrichées servant de réserve de bois pour l'exploitation principale, si bien que cette façon de procéder ne lèse pas davantage les cohéritiers. 
TABLEAU 10

Mode de transmission des terres additionnelles

selon le mode de transmission de l'exploitation principale

\begin{tabular}{|c|c|c|c|c|c|c|c|c|c|c|}
\hline \multirow{2}{*}{$\begin{array}{l}\text { Mode de trans- } \\
\text { mission de } \\
\text { l'exploitation } \\
\text { principale }\end{array}$} & \multicolumn{9}{|c|}{ Mode de transmission des terres additionnelles } & \multirow[t]{2}{*}{ Total } \\
\hline & Partage & $\begin{array}{c}\text { Partage } \\
\text { et } \\
\text { donation }\end{array}$ & $\begin{array}{c}\text { Partage } \\
\text { et } \\
\text { vente }\end{array}$ & $\begin{array}{l}\text { Donation } \\
\text { à rente }\end{array}$ & $\begin{array}{c}\text { Donation } \\
\text { en } \\
\text { avancement } \\
\text { d'hoirie }\end{array}$ & $\begin{array}{l}\text { Vente } \\
\text { à un } \\
\text { fils }\end{array}$ & $\begin{array}{c}\text { Donation } \\
\text { avec } \\
\text { paiement } \\
\text { de } \\
\text { légitime }\end{array}$ & $\begin{array}{c}\text { Donation } \\
\text { avec } \\
\text { renonciation } \\
\text { à la } \\
\text { succession }\end{array}$ & Cession & \\
\hline Partage & $5(5)$ & & & & 5 & 3 & & 1 & & 14 \\
\hline $\begin{array}{l}\text { Partage et } \\
\text { donation }\end{array}$ & 4 & $10(7)$ & 1 & 4 & 8 & 2 & & 1 & 2 & 32 \\
\hline Donation & & & 1 & $13(5)$ & 23 & 5 & 1 & 6 & & 49 \\
\hline Total & $9(5)$ & $10(7)$ & 2 & $17(5)$ & 36 & 10 & 1 & 8 & 2 & 95 \\
\hline
\end{tabular}


Le plus souvent, cependant, les parents destinent ces terres additionnelles à un autre bénéficiaire que celui de la terre principale. La façon la plus courante de procéder est la donation en avance d'hoirie faite au contrat de mariage ou dans un acte spécifique. Ces donations précisent que le bénéficiaire devra rendre compte de la valeur de la terre à ses cohéritiers. Plus rarement, le donataire renonce à la succession de ses parents. Ce procédé est surtout utilisé par les veufs remariés pour désintéresser leurs enfants du premier lit. Certains des transferts de propriété entre parents et enfants prennent la forme d'une vente dont le prix est payable aux cohéritiers. Une donation prévoit le paiement de légitimes. Deux cessions ne précisent ni obligation de retour ni prix de vente, mais dans les deux cas, elles portent sur des terres non défrichées.

Certaines des terres additionnelles font l'objet d'une donation à rente à un autre donataire que celui de la terre principale (12 terres). Ce n'est qu'exceptionnellement que cette pratique lèse les autres héritiers, car la plupart du temps, lorsque les parents recourent à ce procédé, ils disposent de l'ensemble de leurs ajouts en les donnant en bloc à tous leurs héritiers ou en en assignant un à chacun d'entre eux.

Dans la plupart des cas, les terres additionnelles données à rente, vendues ou données en avance d'hoirie n'introduisent pas d'inégalités entre les héritiers. Elles contribuent plutôt à faciliter le dégrèvement de la terre principale pour l'héritier qui s'y est établi ${ }^{20}$. Plus ces terres additionnelles ont de la valeur, moins ce dernier a à payer pour dédommager ses cohéritiers.

\section{3 - La transmission des meubles par les contrats de mariage et les testaments}

$\mathrm{Si}$, le plus souvent, l'aliénation des terres additionnelles à certains enfants avant le décès des parents ne crée pas d'inégalités, qu'en est-il des donations de meubles ou d'argent faites au moment du mariage? Sans être généralisés, de tels dons sont faits dans bon nombre de familles, surtout chez les plus aisées ${ }^{21}$. On donne aux garçons «deux boeufs, une vache, un cochon, un mouton» alors que les filles reçoivent «un lit garny, un buffet, une vache, une brebis et un petit rouet», plus rarement des espèces sonnantes ${ }^{22}$. Mais, dans tous les cas, les bénéficiaires doivent tenir compte de ces avances à leurs cohéritiers.

20 L. Dechêne, Habitants et marchands..., op. cit., 297.

21 Ce jugement basé sur l'examen des contrats de mariage doit être nuancé. Les avances faites au moment du mariage n'y apparaissent pas toujours, comme le prouve la déclaration des 5 héritiers de Pierre Payet et de Catherine Demers qui stipule que chacun a reçu lors de son mariage $258 \#$ «en habits, linges, bestyaux et ustancilles de ménage et ce en avancement d'hoirie». Or, aucun des 5 contrats de mariage ne mentionne ces avances. ANQM, M.not. C.-F. Coron, 20 mars 1743, Déclaration des héritiers Payet.

22 ANQM, M.not. Soupras, 17 janvier 1767, Contrat de mariage de Philippe Bélanger et de Marie Joseph Lauson; M.not. C.-F. Coron, 3 janvier 1745, Contrat de mariage de Joseph Labelle et de Louise Levannier. 
Les testaments ne constituent pas non plus une façon d'avantager un héritier. Peu nombreux (seulement 5 pour les 118 ménages), ils sont le plus souvent le fait de parents âgés ayant déjà disposé du gros de leurs biens et qui désirent régler certaines questions de nature spirituelle et donner les quelques vêtements et meubles qui leur restent. Ces testaments sont d'ailleurs à peu près le seul lieu où transparaissent des préférences affectives de la part des parents. Particulièrement touchant est le souci que met Jean-François Labelle à ne pas laisser démuni son fils Joseph qui est «fort incommodé, n'ayant qu'une jambe». Le testament prévoit qu'en plus de sa part successorale, ce dernier aura 3 perches de terre de plus que les autres enfants et que $100 \#$ seront prises sur les biens de son père pour payer son apprentissage chez un cordonnier $^{23}$.

\section{4 - ÉGALITÉ OU INÉGALITÉ: LA SITUATION AU-DELÀ DES PRATIQUES}

Au terme de cette observation des modes de transmission, peut-on risquer un bilan du degré d'égalitarisme des pratiques observées? L'entreprise est délicate car des éléments de certains dossiers familiaux m'ont certainement échappé. Je pense ici à ce qui est susceptible de ne pas passer devant notaire, mais également au fait qu'il est parfois difficile de juger si deux terres se valent ou si les légitimes correspondent bien à la moitié de ce que les héritiers auraient reçu advenant un partage égalitaire. Mais, même si les résultats sont sujets à caution, l'expérience vaut la peine d'être tentée. En établissant des catégories assez larges, les risques d'erreurs sont d'ailleurs atténués (tableau 11).

Ce que révèle ce bilan, c'est qu'à l'île Jésus entre 1720 et 1775 , on est encore loin de la transmission du patrimoine à un seul bénéficiaire. L'égalitarisme total est le fait d'une minorité de familles, mais seulement 11 successions ne prévoient rien ou très peu pour les héritiers désavantagés. Par contre, dans 104 des 118 cas, ceux-ci ont touché au moins leur légitime.

La situation fait néanmoins des perdants et les principales sont les filles qui reçoivent rarement plus qu'une légitime en argent. Sauf dans les cas de partage et dans ceux où la donation est faite à l'ensemble des enfants, elles sont exclues de la transmission des biens fonciers car, à deux exceptions près, elles ne reçoivent pas de terre en avance d'hoirie.

Comme le tableau 11 le montre, la généralisation de la donation qui apparaît au milieu du XVIIIe siècle ne signifie pas toujours l'exclu-

23 Avant de le placer chez un cordonnier de l'île Jésus, Labelle avait engagé Joseph en tant qu'apprenti chez un tailleur d'habit de Montréal. C'est dans cet engagement qu'on apprend la nature de son infirmité. ANQM, M.not. J.B. Adhémar, 8 mars 1741, Engagement de Joseph Labelle à Vincent Morant; M.not. C.-F. Coron, 29 septembre 1741, Engagement de Joseph Labelle à Pierre Labrêche; M.not. C.-F. Coron, 27 décembre 1742, Testament de Jean-François Labelle. 
TABLEAU 11

Part des héritiers «défavorisés»

selon le mode de transmission de la terre principale

(118 ménages paysans)

$$
\text { 1720-1775 }
$$

\begin{tabular}{|c|c|c|c|c|c|c|c|c|}
\hline \multirow{2}{*}{\multicolumn{2}{|c|}{$\begin{array}{ll} & \begin{array}{l}\text { Mode de } \\
\text { transmission }\end{array} \\
\text { de la terre } \\
\text { principale } \\
\end{array}$}} & \multicolumn{5}{|c|}{ Part des «défavorisés»* } & \multirow{2}{*}{$\begin{array}{c}\text { sans } \\
\text { défavorisés }\end{array}$} & \multirow[t]{2}{*}{$\begin{array}{c}\text { Total } \\
\text { Successions }\end{array}$} \\
\hline & & Rien & $\begin{array}{c}\text { somme } \\
\text { symbolique }\end{array}$ & $25 \%-49 \%$ & $50 \%-74 \%$ & $75 \%-99 \%$ & & \\
\hline 珞 & Partage & & & & & & 20 & 20 \\
\hline 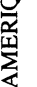 & $\begin{array}{l}\text { Partage } \\
\text { et vente }\end{array}$ & & & & $3 * *$ & $3 * *$ & & 6 \\
\hline 可 & $\begin{array}{l}\text { Partage et } \\
\text { donation }\end{array}$ & & & 1 & 28 & 16 & 4 & 49 \\
\hline 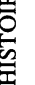 & $\begin{array}{l}\text { Donation } \\
\text { à rente }\end{array}$ & 7 & 3 & 2 & 23 & 4 & 3 & 42 \\
\hline 勻 & Renonciation & 1 & & & & & & 1 \\
\hline 空 & Total & 8 & 3 & 3 & $54 * *$ & $23 * *$ & 27 & 118 \\
\hline
\end{tabular}


sion complète des héritiers non donataires. Cette évolution de la pratique n'est cependant pas négligeable parce qu'elle signifie un traitement différent des héritiers selon le sexe.

\section{5 - LES FACTEURS ORIENTANT LES PRATIQUES DE TRANSMISSION}

Que peut-on conclure de l'observation de ces pratiques? L'examen attentif des cas où il y a donation montre que le recours à cette forme de transmission n'a vraisemblablement pas pour but de favoriser un héritier au détriment des autres. Elle permet plutôt aux parents d'atteindre deux objectifs partiellement contradictoires: établir le mieux possible le plus de fils possible et satisfaire le désir bien légitime d'assurer leur retraite.

Ce dernier objectif est souvent oublié lorsqu'on discute de l'orientation des systèmes de reproduction familiale. N'ayant à l'esprit que la finalité de la reproduction, on considère que ces systèmes oscillent entre deux extrêmes: d'un côté, l'exclusion des non-héritiers dans le but de préserver l'intégrité du patrimoine et, de l'autre, l'établissement du plus grand nombre d'enfants possible et, pour ce faire, l'expansion de la propriété. Pourtant, en transmettant de leur vivant, il est clair que les parents cherchent également à assurer leurs vieux jours.

Ce but ne peut cependant être atteint que si la terre principale du couple a une capacité productive suffisante pour subvenir aux besoins matériels d'au moins deux couples et des enfants à venir. Dans le terroir que nous avons étudié, certaines exploitations ont des rendements assez élevés pour, d'une part, faire vivre le ménage du donataire et permettre le paiement d'une rente et, d'autre part, assurer des légitimes valables aux cohéritiers. Le recours à la donation devient alors possible.

On peut donc en conclure que le recours à la donation qui apparaît au milieu du XVIIIe siècle n'est sans doute pas d'abord attribuable à la disparition des valeurs égalitaires de la période pionnière. Elle est plutôt la conséquence de la mise en valeur progressive des exploitations. Les paysans propriétaires d'exploitations fournissant de bons surplus sont en mesure, d'une part, d'étendre leur patrimoine foncier par l'achat de terres additionnelles pendant la jeunesse de leurs enfants de façon à en établir le plus possible et, d'autre part, de se procurer une retraite en faisant donation d'une exploitation capable de faire vivre plus d'un ménage. À ce titre, il est intéressant de constater que les parents qui recourent à la donation pour transmettre la terre principale sont ceux qui disposent du plus grand nombre de terres additionnelles (tableau 10). On doit donc se méfier du psychologisme sommaire souvent attaché aux études de la transmission. Avant de déduire de 
l'observation des pratiques, l'existence de valeurs égalitaires ici et de valeurs inégalitaires là, il faut prendre en considération tout le contexte de la transmission tant économique que juridique ${ }^{24}$.

Par ailleurs, les résultats de cette enquête montrent que l'adoption progressive de la donation ne découle pas de la saturation du terroir. l'île Jésus, en effet, les donations commencent à se multiplier au moins trente ans avant la fin de la colonisation de l'île et presque soixante-quinze ans avant que le peuplement des seigneuries situées directement au nord ne soit complété.

D'ailleurs, la situation qu'on retrouve à l'île Jésus au milieu du XVIIIe siècle correspond, en grande partie, aux pratiques de transmission décrites par Gérard Bouchard comme typiques d'un «système ouvert» de reproduction familiale.

Cependant cette combinaison donation/attribution de terres additionnelles n'apparaît pas d'abord liée au contexte de colonisation. En effet, pour adopter cette forme de transmission, les paysans doivent en avoir les moyens. Si l'objectif est l'établissement réel d'un maximum de descendants, il faut que les «ajouts» attribués lors de la première phase de transmission décrite par Gérard Bouchard soient des terres défrichées. Pour être en mesure de transmettre de telles terres, les parents ont deux possibilités: l'achat de lots déjà défrichés ou l'acquisition et la mise en valeur de terres en bois debout. La première stratégie requiert évidemment de l'argent, mais la seconde n'est pas non plus à la portée de tous les paysans. Au XVIIIe siècle, les terres non défrichées sont concédées gratuitement, mais les seigneurs ne sont pas très favorables à l'accumulation de tenures non exploitées que cette pratique suppose et plusieurs paysans ne sont pas en mesure d'assumer pendant de longues années le poids des diverses charges qui pèsent sur ces terres peu productives (redevances seigneuriales, répartition paroissiale, corvée de voirie) ${ }^{25}$. Par ailleurs, au XIXe siècle, les terres de colonisation doivent être achetées. Enfin, dans les deux cas, il faut que les couples soient capables de défrayer les coûts qu'entraîne le défrichement. Bref, pour pouvoir consacrer temps et argent à l'acquisition et à la mise en valeur de terres additionnelles et ainsi établir les non-donataires, les couples doivent déjà posséder une bonne exploitation agricole. Sans cela, ils transmettent par donation en excluant les non-donataires ou ils se contentent de garder leurs biens qui seront, après leur décès, partagés égalitairement, comme c'était le cas au tout début de la période de colonisation.

24 E. P. Thompson, «The Grid of Inheritance: a Comment», J. Goody, J. Thirsk et E. P. Thompson, eds., Family and Inheritance, Rural Society in Western Europe 1200-1800 (Londres, Cambridge University Press, 1976), 342. Cette exigence a été récemment démontrée pour la France d'Ancien Régime par Bernard Derouet, «Pratiques successorales et rapport à la terre: les sociétés paysannes d'Ancien Régime», Annales ESC, 44,1 (janv.-fév. 1989): 173-206.

${ }_{25}$ Sylvie Dépatie, «La structure agraire au Canada: le cas de l'île Jésus au XVIIIe siècle», Communications historiques (Winnipeg, 1986), 81. 
La donation de l'exploitation principale et l'établissement du plus grand nombre possible d'héritiers par l'attribution de terres acquises du vivant des parents ne dépend donc pas d'abord de la disponibilité de terres libres. Pour que cette pratique soit efficace, c'est-à-dire pour qu'elle procure un établissement valable aux non-donataires, elle requiert, en effet, une certaine aisance économique de la part des parents et donc la possession d'une exploitation agricole suffisamment mise en valeur pour produire des surplus ${ }^{26}$. Sans cette condition, même en terroir neuf, il est impossible d'établir des enfants par l'expansion de la propriété.

\section{6 - L'EFFICACITÉ DE LA TRANSMISSION ET LA REPRODUCTION SOCIALE DE LA CELLULE FAMILIALE}

Jusqu'ici, ce sont les pratiques de transmission et leur degré d'égalitarisme qui ont retenu notre attention. Mais, dans le cadre de cette étude, il est tout aussi important d'en cerner l'efficacité. Cette notion peut différer selon qu'on se place du côté des parents ou de celui des enfants. Pour les premiers, une transmission réussie peut être celle qui garde intact un bien familial se transmettant de générations en générations ${ }^{27}$. Cependant, l'étude des pratiques des paysans aisés de l'île Jésus laissent plutôt voir que, tout en cherchant à préserver l'intégrité de l'exploitation pour s'assurer une rente viagère, les parents cherchent aussi à établir du mieux qu'ils le peuvent le plus grand nombre d'enfants possible. Ces derniers souscrivent certainement à cet objectif, de sorte qu'on peut dire que l'efficacité du système de transmission se mesure en fonction de sa capacité à faire accéder les enfants à la propriété de la terre productive. En regard de cet objectif, il est possible, à l'instar de Louis Michel qui a étudié le phénomène à Varennes et à Verchères, d'envisager quatre situations.

La première est la «reproduction élargie parfaite» de la cellule familiale où, par l'achat et la mise en valeur de terres additionnelles, les parents réussissent à établir tous les garçons. La seconde est la «reproduction élargie partielle» par laquelle seulement certains fils sont établis. La troisième est la «reproduction simple» où les parents n'arrivent à établir qu'un seul des garçons, au détriment des autres, en lui donnant la seule terre qu'ils possèdent. Enfin, la quatrième situation, «l'absence de reproduction», est celle où les parents n'établissent

26 Étudiant la transmission du patrimoine dans le Haut Saint-Jean au XIXe siècle, Béatrice Craig rejette également le degré de saturation du terroir comme facteur unique de l'adoption de la donation. Elle y substitue l'existence d'un large éventail d'occupations pour les exclus, phénomène inexistant au XVIIIe siècle dans la région présentement étudiée mais dont il faut absolument tenir compte lorsqu'il apparaît. B. Craig, «La transmission des patrimoines fonciers dans le Haut Saint-Jean au XIXe siècle», communication non publiée, présentée au Congrès de la Société historique du Canada, Québec, juin 1989.

27 E. Le Roy Ladurie, "Système de la Coutume, structures familiales et coutume d'héritage en France au XVIe siècle», Annales ESC, 27,4-5 (juillet-octobre 1972): 828-829. 
aucun de leurs enfants. Dans les trois derniers cas, le processus de transmission crée des exclus qui ne reçoivent pas de terre. Ceux-ci deviennent journaliers ou prennent en concession des terres en bois debout dans le terroir local ou à l'extérieur dans des terroirs de colonisation plus récente ${ }^{28}$.

Ajoutons, avec Louis Michel, que la réalité n'est pas toujours aussi tranchée. Même s'ils ne reçoivent pas de terre, certains héritiers touchent une somme d'argent plus ou moins importante qui facilite leur installation sur une terre neuve, voire qui leur permet d'acheter une terre dont la mise en valeur est un peu plus avancée. Par contre, dans les cas de reproduction élargie, les terres fournies aux fils peuvent ne pas avoir la même valeur, si bien que certains sont mieux établis que d'autres. Les quatre situations décrites plus haut peuvent donc être multipliées en une vaste gamme qui va de la réussite totale et certainement utopique, où tous les fils reçoivent une terre aussi développée que la terre principale, à l'échec total et sans doute beaucoup plus probable, où aucun des garçons n'a accès à la propriété.

Il n'est évidemment pas possible de tenir compte de toutes ces nuances pour mesurer l'efficacité de la transmission des 118 famillestémoins. Leur réussite est donc évaluée en fonction des quatre situations décrites plus haut (tableau 12) ${ }^{29}$. Précisons tout de suite la fragilité de ce classement qui tient à la complexité des situations en présence, aux lacunes documentaires et à l'ambiguiité même du concept d'établissement. Celui-ci ne peut être restreint à l'achat de terres défrichées par les parents à l'intention des héritiers. Une bonne légitime ou le produit de la vente de parts successorales peut servir à l'achat d'une telle terre directement par l'héritier. Or, les sources ne permettent pas toujours de vérifier si tel a été le cas, pas plus d'ailleurs qu'elles ne permettent de déterminer la part prise par les parents dans l'établissement d'un fils qui, encore jeune, obtient par concession une terre non défrichée. Il faut donc compenser ces lacunes documentaires par des jugements qualitatifs basés sur une bonne connaissance des dossiers familiaux. Par ailleurs, en raison de la composition de la populationtémoin, nos résultats sous-estiment grandement le taux d'échec du

28 L. Michel, "Varennes et Verchères, des origines au milieu du XIXe siècle: état d'une enquête», J. Goy et J.-P. Wallot, dir., Évolution et éclatement du monde rural. Structures, fonctionnement et évolution différentielle des sociétés rurales françaises et québécoises XVIIe-XXe siècles (Paris/Montréal, Éditions de l'École des Hautes Études en Sciences Sociales/Presses de l'Université de Montréal, 1986), 325-340. Actes du colloque franco-québécois d'histoire rurale comparée de Rochefort, 5-8 juillet 1982.

29 Cette mesure de l'efficacité en fonction de la capacité d'établir l'ensemble des fils d'un ménage ne recouvre pas toutes les situations de notre échantillon. La "performance» des familles ne comptant que des filles a été évaluée en fonction du nombre de filles. Celle des familles comptant des fils mais où la donation est faite à un gendre ou à un étranger a été évaluée comme si le ou les donataires étaient des membres de la famille en fonction du nombre de fils à établir. Cette procédure a été adoptée parce que c'est avant tout la capacité d'établir qui nous intéresse. 


\section{TABLEAU 12}

Mesure de l'efficacité de la transmission selon le mode de transmission de l'exploitation principale

(118 ménages paysans)

1720-1775

\begin{tabular}{|c|c|c|c|c|c|c|}
\hline \multirow{2}{*}{$\begin{array}{l}\text { Mode de } \\
\text { transmission } \\
\text { de la terre } \\
\text { principale }\end{array}$} & \multicolumn{5}{|c|}{ Efficacité de la transmission } & \multirow[t]{2}{*}{ Total } \\
\hline & $\begin{array}{l}\text { Absence de } \\
\text { reproduction }\end{array}$ & $\begin{array}{l}\text { Reproduction } \\
\text { simple }\end{array}$ & $\begin{array}{c}\text { Reproduction } \\
\text { élargie } \\
\text { partielle }\end{array}$ & $\begin{array}{c}\text { Reproduction } \\
\text { élargie } \\
\text { parfaite }\end{array}$ & Indéfinie & \\
\hline Partage & 7 & 8 & 2 & 3 & & 20 \\
\hline $\begin{array}{l}\text { Partage } \\
\text { et vente }\end{array}$ & 5 & 1 & & & & 6 \\
\hline $\begin{array}{l}\text { Partage et } \\
\text { donation }\end{array}$ & 0 & 33 & 11 & 4 & 1 & 49 \\
\hline Donation & 0 & 19 & 7 & 16 & & 42 \\
\hline Renonciation & 1 & & & & & 1 \\
\hline Total & 13 & 61 & 20 & 23 & 1 & 118 \\
\hline
\end{tabular}

Sources: Actes notariés et fichier des ménages paysans. 
processus de transmission. En effet, dans bon nombre de cas où il y a absence de reproduction, les parents n'arrivent pas à établir leurs enfants parce que, trop endettés, ils ont dû vendre leur exploitation avant de pouvoir la transmettre ${ }^{30}$. Or, les familles que nous étudions avaient toutes au moins une terre à transmettre, ce qui réduit la proportion des cas où aucun héritier n'est établi et rend certainement moins dramatique le destin des exclus.

En dépit de cela, il y a absence de reproduction dans plus du dixième des cas observés. Il s'agit d'exemples où la terre paternelle a échappé aux héritiers lors du processus de transmission, en les laissant sans ressources ou avec des moyens trop faibles pour acheter des terres constituant un établissement valable.

Dans la majorité des familles-témoins, la terre paternelle reste cependant aux mains d'un des héritiers, mais la plupart du temps, celuici est le seul à être établi. À peine plus du tiers des couples arrivent à pourvoir plus d'un fils, mais le plus souvent ils ne sont pas en mesure de tous les établir. En définitive ce n'est qu'une minorité de familles qui ont été en mesure de pourvoir à l'établissement de tous leurs garçons (tableau 12).

À première vue, cette inégalité dans la capacité de reproduire la cellule familiale peut être attribuée au fait que tous ces ménages n'avaient pas le même nombre de fils à établir. Il est en effet logique de penser que les familles comptant moins de fils étaient plus susceptibles de réussir une reproduction élargie parfaite que les familles comptant un grand nombre de descendants mâles. Pour minimiser l'influence de ce facteur, somme toute accidentel, j'ai classé comme cas de reproduction simple les 17 familles qui n'avaient qu'un fils à établir. Cependant l'incidence du nombre de fils à établir sur le succès de la reproduction ne doit pas être écartée. Pour la mesurer, j'ai calculé le nombre moyen de fils pour chacun des degrés d'efficacité de la reproduction (tableau 13). Ce calcul montre que le nombre de fils à établir n'a pas une influence déterminante sur le succès de la reproduction. Le fardeau plus lourd que constitue la présence de nombreux fils est sans doute partiellement compensé par l'avantage d'une capacité de travail accrue dans les années où la famille travaille à l'établissement des garçons.

Les hasards de la démographie jouent cependant un rôle dans la réussite ou l'échec de la reproduction. Ainsi, la plupart des cas d'absence de reproduction sont le fait de familles où le père est disparu prématurément en laissant un bien foncier de faible valeur. Ces couples n'ont donc pas eu la possibilité de développer une exploitation susceptible de fournir un point de départ valable à leurs héritiers et ils n'ont

30 L. Michel, loc. cit., 333. 
pas pu acheter des terres additionnelles pour faciliter le processus de reproduction.

\section{TABLEAU 13}

Nombre moyen de fils

selon l'efficacité de la transmission

(118 ménages paysans)

1720-1775

\begin{tabular}{lcc}
\hline \multicolumn{1}{c}{$\begin{array}{c}\text { Efficacité de } \\
\text { la transmission }\end{array}$} & $\begin{array}{c}\text { Nombre } \\
\text { de cas }\end{array}$ & $\begin{array}{c}\text { Nombre moyen } \\
\text { de fils }\end{array}$ \\
\hline $\begin{array}{l}\text { Absence de } \\
\text { reproduction }\end{array}$ & 13 & 2.5 \\
$\begin{array}{l}\text { Reproduction } \\
\text { simple }\end{array}$ & 61 & 2.9 \\
(ensemble des cas) & 44 & 3.6 \\
$\begin{array}{l}\text { Reproduction } \\
\text { simple } \\
\text { (cas comptant plus }\end{array}$ & 20 & \\
d'un fils) & 23 & 4.6 \\
$\begin{array}{l}\text { Reproduction } \\
\text { élargie partielle }\end{array}$ & 1 & 3.0 \\
$\begin{array}{l}\text { Reproduction } \\
\text { élargie parfaite }\end{array}$ & & 3.0 \\
\hline Indéfinie & & \\
\hline
\end{tabular}

Sources: Actes notariés et fichier des ménages paysans.

Ce fait est important car, en définitive, l'inégalité dans la capacité de reproduire efficacement la cellule familiale tient en bonne partie au fait que certains couples achètent et mettent en valeur des terres additionnelles alors que d'autres ne transmettent qu'une seule terre. Or, la capacité de grossir le patrimoine foncier est très influencée par la situation de départ des couples. Ceux qui commencent leur vie adulte sur une terre en bois debout doivent réserver leur temps et leur capital à développer celle-ci tandis que ceux qui, à la même étape, sont déjà à la tête d'une bonne exploitation, pas trop grevée de dettes envers leurs cohéritiers, peuvent plutôt consacrer temps et argent à l'acquisition et à la mise en valeur de terres additionnelles. Quand, à leur tour, ils commencent à transmettre leur patrimoine, ces individus ont plus de terres. Cela leur permet d'établir plus d'enfants, d'alléger le fardeau de celui qui reprend l'exploitation principale et de se montrer plus égalitaires que d'autres qui, n'ayant qu'une terre, doivent se résoudre à la transmettre à un seul héritier en laissant les autres plus ou moins démunis. 
Tout ceci explique que, dans un contexte de transmission relativement égalitaire, les héritiers de certaines familles reçoivent plus que ceux d'autres familles. Plus que le degré d'égalité ou d'inégalité, c'est en effet la taille du patrimoine familial qui détermine la réussite du processus de transmission et le sort de la génération subséquente.

\section{7 - LA REPRODUCTION SOCIALE DE LA CELLULE FAMILIALE ET L'ÉVOLUTION DE LA SOCIÉTÉ PAYSANNE}

Le degré d'égalité dans les pratiques de transmission n'est cependant pas une question d'érudition réservée aux adeptes de la psychologie collective. Puisque la transmission du patrimoine est le mécanisme central de la reproduction de la société rurale, ses modalités ont une influence déterminante sur l'évolution de la société.

Ce que révèle l'étude des pratiques de transmission à l'île Jésus, c'est qu'en dépit d'un recours fréquent à la donation, le souci d'installer les enfants non donataires existe et se manifeste par les légitimes et la distribution en leur faveur des terres additionnelles. La presque totalité des transmissions analysées donne donc lieu à une redistribution, au moins partielle, des avoirs familiaux. La conséquence de cette redistribution qui se répète à chaque génération est bien connue: elle défavorise l'accumulation foncière, gểne le développement du capitalisme agraire en créant un endettement permanent et renforce les bases de l'économie familiale ${ }^{31}$. L'héritier établi sur l'exploitation familiale doit la racheter en tout ou en partie de ses cohéritiers, ce qui l'empêche d'investir dans son développement. Quant aux héritiers défavorisés, ils doivent s'installer sur des terres de moindre valeur, souvent même sur des terres non défrichées.

Le système de transmission explique donc deux phénomènes observés à l'île Jésus et ailleurs au Canada au XVIIIe siècle, soit la lenteur de la mise en valeur des terres et la présence continuelle d'exploitations très peu défrichées, n'ayant qu'une faible capacité productive $^{32}$. Ce dernier trait est d'ailleurs plus accentué que ne le laisse voir l'observation limitée aux frontières de l'île car, dès 1740 , les exclus de la transmission essaiment vers le nord dans les seigneu-

31 L. Dechêne, Habitants et marchands..., op. cit., 298; G. Bouchard, «Sur la reproduction familiale en milieu rural...», loc. cit., 243.

32 S. Dépatie, L'évolution d'une société rurale..., op. cit., 197-212, 233-260. Ces caractéristiques observées à l'île Jésus sont signalées dans d'autres régions rurales du Québec à la même époque. Pour les rythmes de défrichement, voir L. Dechêne, op. cit., 273-278 et J. SaintPierre, "L'aménagement de l'espace rural en Nouvelle-France: les seigneuries de la Côte du sud», J. Mathieu et S. Courville, Peuplement colonisateur aux XVIIe et XVIIIe siècles (Québec, CÉLAT, Université Laval, coll. «Cahiers du Célat», no 8, 1987), 113-136. Pour la taille des exploitations, voir C. Dessureault, Les fondements de la hiérarchie sociale au sein de la paysannerie: le cas de Saint-Hyacinthe, 1760-1815, thèse de Ph.D. (histoire), Université de Montréal, 1985, 315-387; et Thomas Wien, Peasant Accumulation in a Context of Colonization, Rivière$d u$-Sud, Canada, 1720-1775, thèse de Ph.D. (histoire) Université McGill, 1988, 218-232. 
ries de Terrebonne et de Mille-Iles et bientôt vers celles de Blainville et de Rivière-du-Chêne où ils s'installent sur des terres neuves. Cette «mise à zéro» est le destin de la plupart des paysans de chaque génération puisque la majorité des cas de transmission produit des exclus.

À l'inverse, le système de transmission égalitaire permet le développement d'unités de production dont la taille est bien au-dessus de la moyenne ${ }^{33}$. Les dossiers familiaux constitués aux fins de cette étude montrent que ces exploitations ne sont pas le résultat temporaire d'un processus d'accumulation foncière précédant une inévitable distribution au moment de la transmission du patrimoine ${ }^{34}$. L'examen des stratégies familiales de transmission montre en effet que le cycle accumulation-distribution n'est pas un processus continu qui débouche sur une redistribution en un seul coup, mais plutôt un processus fragmenté à l'intérieur duquel les acquisitions de terre n'interviennent souvent qu'au moment de l'établissement du fils auquel on la destine.

Dès lors, l'existence de grosses exploitations s'explique plutôt par le fait que, même dans un contexte de relative égalité, une minorité de familles arrivent à ce qu'aucun des héritiers ne soit refoulé vers la périphérie. Leurs pratiques de transmission comportent une certaine égalité et entraînent donc une redistribution des avoirs familiaux, mais l'importance du patrimoine familial leur permet tout de même d'établir la plupart de leurs fils sur des terres dont la mise en valeur est très avancée. Ayant accès à moindre coût et plus tôt à la terre productive, ces héritiers de familles aisées sont en mesure de mieux développer leurs exploitations que ne le peuvent la plupart des paysans qui commencent leur vie active sur une terre en bois debout ou sur une terre plus développée mais lourdement grevée de dettes successorales.

\section{CONCLUSION}

L'étude des pratiques de transmission à l'île Jésus a permis de confirmer que le milieu du XVIIIe siècle est marqué par la généralisation de la donation. Cette pratique conduit rapidement à l'exclusion des filles de la transmission des biens fonciers mais elle ne signifie pas nécessairement l'inégalité pour les garçons. Telle qu'utilisée par les familles étudiées, la donation permet plutôt aux parents de concilier deux objectifs contradictoires, soit la perception d'une rente viagère et l'établissement d'un maximum de fils, par le biais de la transmission de terres additionnelles.

\footnotetext{
33 Voir note précédente.

34 À cet égard, je rejoins les conclusions de Christian Dessureault, Les fondements..., op. cit. , 444-494; "L'égalitarisme dans l'ancienne société rurale de la vallée du Saint-Laurent», Revue d'histoire de l'Amérique française, 40,3 (hiver 1987): 373-407, et de Thomas Wien, op. cit., 227-229.
} 
Plutôt que d'être le fruit de la saturation du terroir, la donation à rente apparaît donc davantage liée à la capacité des exploitants de générer des surplus essentiels au paiement de la rente viagère, au versement de légitimes et à l'acquisition de terres additionnelles. La généralisation de cette pratique est, par conséquent, conditionnée par la mise en valeur progressive du terroir et par l'aisance économique des paysans qui l'adoptent. Il est clair, en effet, que la nature des biens transmis influence le choix des formes de transmission et que, pour comprendre ces dernières, il faut tenir compte du contexte économique dans lequel elles s'exercent.

Par ailleurs, la mesure de l'efficacité de la reproduction montre que l'égalitarisme en matière de transmission ne conduit pas au nivellement des fortunes mais que, au contraire, il permet, voire accentue, les écarts économiques à chaque génération.

Enfin, il est évident que la présence de terres disponibles à proximité influence les modalités de la transmission, mais qu'elle ne contribue en rien à augmenter l'efficacité du système de reproduction sociale, si on entend par efficacité autre chose que le maintien d'une société paysanne dont la survivance implique, à chaque génération, la marginalisation d'une majorité de ses membres. 